\title{
Knowledge Graph Construction for Intelligent Media Based on Mobile Internet
}

\author{
Jianhua Dai 1 and Jingxin $\mathrm{Xu}$ \\ Business School, China University of Political Science and Law, Beijing 100088, China \\ Correspondence should be addressed to Jianhua Dai; daijianhua66@163.com
}

Received 15 October 2021; Revised 16 November 2021; Accepted 6 December 2021; Published 11 January 2022

Academic Editor: Ming Yan

Copyright (C) 2022 Jianhua Dai and Jingxin Xu. This is an open access article distributed under the Creative Commons Attribution License, which permits unrestricted use, distribution, and reproduction in any medium, provided the original work is properly cited.

\begin{abstract}
Mobile Internet-based intelligent media has become a popular academic topic. This study uses the CiteSpace visualisation tool and Scientific Citation Index Expanded database to comb the existing research in the field of intelligent media from a quantitative perspective. A total of 7248 English papers were published on the topic of "intelligent media" from 2012 to 2021 , and 145 highly cited papers refined were analysed. Scientific knowledge graphs were analysed from six dimensions: annual publication quantity, country of publication, institution of publication, author, keywords, and cited references. In the last 10 years, the research literature on intelligent media has been found to increase annually. Presently, the People's Republic of China and the United States of America have a high proportion of documents in this field. Chinese universities and institutions have achieved significantly in terms of the quantity and quality of documents. From the perspective of the whole intelligent media discipline, the high-yield author group has not been formed, and there is minimal cooperation amongst authors. Popular intelligent media topics include film, social media, machine learning, swarming motility, data mining, and artificial intelligence. Subject words of the main research directions are event recognition, fake news, Cable News Network model, reconfigurable intelligent surface, comprehensive survey, microblog message, strain sensor, and traffic event. Combined with popular topics and time zone maps, the future research frontier in the field of smart media is identified.
\end{abstract}

\section{Introduction}

Presently, the development of mobile communication, artificial intelligence, intelligent terminal technology, and social media has resulted in some technologies penetrating the media industry, such as Internet of things (IoT), cloud computing, virtual augmented reality, intelligent hardware, and big data. The transformation of new media has led to the evolution and change of media forms, content production, distribution channels, user experience, and other fields. The media space dominated by people in the past has been substantially expanded, and intelligent media has gradually developed. With the support of mobile Internet and big data technology, meeting the personalised needs and customised requirements of users has become a possibility. The emer- gence of various media forms has resulted in intelligent media becoming an important development direction for media organisations to win against competitors. Additionally, changes in user behaviour have promoted the intelligent development of media.

In the last decade, the number of intelligent media research has been increasing, and the distribution of intelligent media research in different disciplines has been expanding. The analysis of the development trend of intelligent technology, influence of intelligent technology on intelligent media, media market environment, and future development trend of intelligent media has an important reference value for regulators to formulate intelligent media policies and for enterprises to choose the development path of intelligent media. Although the development 
of intelligent media has provided substantial convenience, it has also brought numerous social problems. This study conducts the necessary examination and reflection on previous academic research.

\section{Literature Review}

According to the search of scholars and Scientific Citation Index Expanded (SCIE) databases, research on intelligent media has mainly focused on such aspects as intelligent technology, personalised recommendation system, user and algorithm credibility, diversity of information environment, participation of intelligent technology in Internet information governance, information values, transparency, and algorithm accountability mechanism. This study selects highly cited articles in the aforementioned fields for analysis.

In the intelligent technology research, Pollack surveyed new technologies that incorporate artificial intelligence techniques to support older adults and help them cope with the changes of aging, particularly with cognitive decline [1]. Brooks et al. provided a detailed description of dataassisted approaches to building intelligent technologyenhanced learning systems, which focus on helping instructional experts discover insights into the teaching and learning process, and leveraged these insights as instructional interventions [2]. Marres evaluated an emerging paradigm for testing intelligent technology in society through the analysis of recent street trials of self-driving cars [3]. Jin et al. proposed the method of combining reward network and cross-entropy loss to guide the training of generators and produce high-quality music works [4]. Zhang et al. provided a computational intelligence method for different system levels, described the development direction of intelligent systems, and introduced the basic state-of-the-art concepts of data mining [5]. For intelligent systems, Liu et al. presented tactile sensors with excellent application prospects in numerous fields, such as wearable devices, medical treatment, artificial limbs, and robotics [6]. Intelligent technology has been widely used in the Industry 4.0 era, in which manufacturing will be via the Internet, to achieve internal and external network integration towards the intelligent direction [7]. Industry 4.0 strategy revolutionises manufacturing systems and processes and also leads to the formation of an intelligent supply chain [8].

In the aspect of a personalised recommendation system, numerous studies have discussed users' preference between personalised and nonpersonalised news. Sang et al. developed a recommendation system that enables bidirectional communication between users and systems using a utility range-based product recommendation algorithm to provide markedly dynamic and personalised recommendations [9]. The traditional recommendation system disregards the internal relationship between user preference and time. To address this issue, Cui et al. proposed a novel recommendation model based on time correlation coefficient and an improved $K$-means with cuckoo search (CSK-means) [10]. When the number of books provided by libraries is relatively high, users experience difficulty selecting the appropriate books from numerous candidate books. For this case, Tian et al. designed a personalised recommendation system for college libraries based on a hybrid recommendation algorithm [11]. According to the characteristics of each user group, Yan et al. proposed an environment prediction algorithm based on mobile user classification, and the algorithm has great application prospects in news recommendation [12]. According to statistics of users in 26 countries, although there are individual differences, users generally believe that the algorithm based on historical consumption behaviour is a better way of news recommendation than manual editing. Such variables as age, trust in news, attention to privacy, and use of mobile news have an impact on this preference [13]. Readers' attitude towards personalised news depends on the diversity of news recommendation systems and degree of care in the field of public news, rather than privacy and other factors [14]. Moreover, users' attitudes towards personalised news are heterogeneous. Young and poorly educated users favour personalised news and have minimal contact with diversified information services provided by public media.

With the help of computer technology, researchers have designed random, manual, and multiple personalised recommendation systems based on the principles of popularity, semantic filtering, and collaborative filtering to measure the impact of recommendation algorithms on content diversity [15]. Accordingly, the diversity of the algorithm recommendation has not been reduced compared with the nonpersonalised recommendation, and content topics recommended by personalised collaborative filtering systems are the most abundant. Nevertheless, the recommendation system continue to face the risk of gradual marginalisation of long-tail content, which should be countered by weighting minority content.

Scholars have determined that the algorithm formulated by dominant organisations can cause the entire industry to follow and reflect the power in the process of controlling the production and circulation of information and also demonstrate dominance by formulating industry norms [16]. Researchers are convinced that apart from emphasising the role of algorithms in constructing reality, people should also realise the function of algorithms as a relationship management mechanism between organisations and individuals [17]. As part of this organisational structure, algorithm systems can automate the management mechanism of leading organisations and also provide a common language or structure to redefine value and affect the legitimacy of other organisations and individuals [18].

In the research of transparency and algorithm accounting mechanisms, fake news remains a difficult problem for Internet users and scholars. The fake news research published by Science magazine has observed the proliferation of all verified real and fake news published on Twitter from 2006 to 2017 and found that the dissemination range of false information substantially exceeds that of real news regardless of theme [19]. Additionally, fake news has proven to have a powerful agenda-setting function [20]. Given the flood of false news and overstretched manual verification 


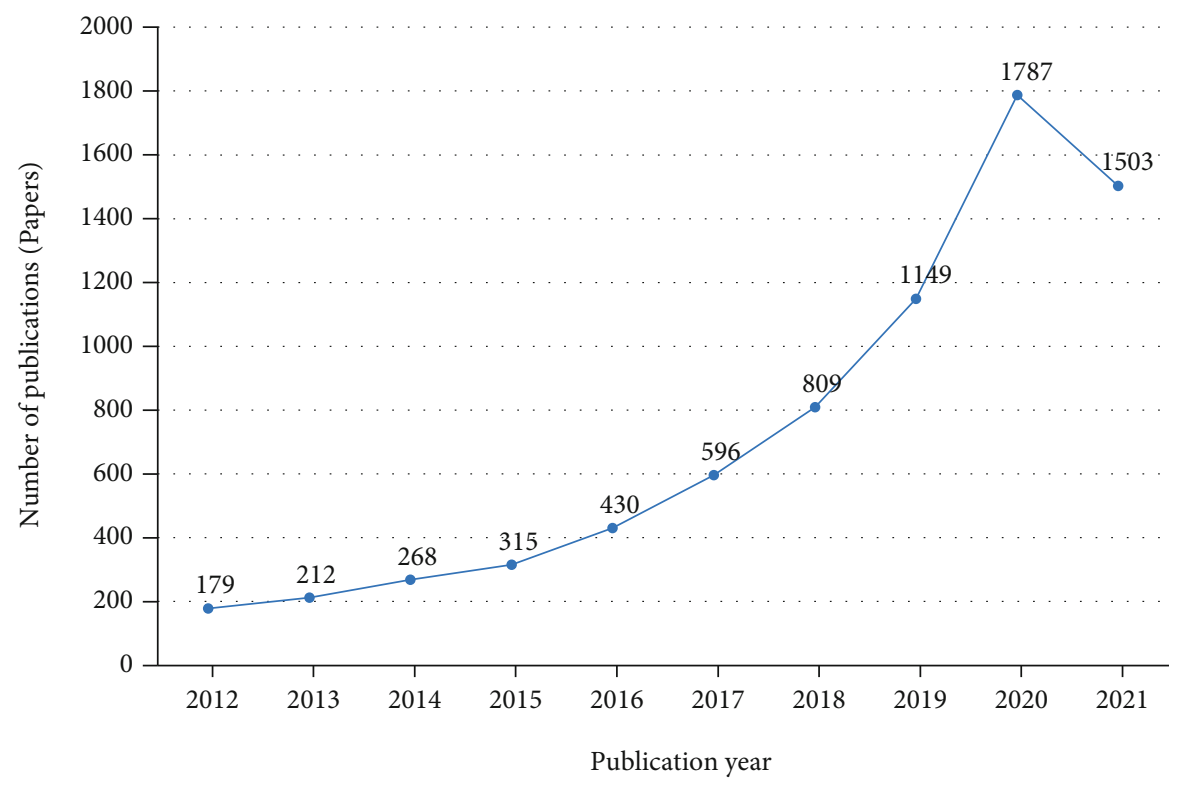

Figure 1: Number of articles published in intelligent media from 2012 to 2021.

in the Internet information environment, intelligent technology has been widely used in identifying and communicating false news [21]. In 2018, Facebook announced changes to its algorithm, prioritising reliable news in response to questions on whether or not its algorithm promotes the social spread of spam [22]. To date, no systematic review has been undertaken of studies conducted in the field of "algorithmic accountability." Wieringa obtained a perspective from critical algorithm research and also provided an interdisciplinary overview using materials from data research to law and from computer science to governance research [23].

\section{Materials, Methods, and Tools}

3.1. Research Tools. This study is based on the research methods of "cocitation analysis" and "cooccurrence analysis" of bibliometrics and conducts visual analysis using CiteSpace software (version 5.8. R3). Mapping knowledge domain is drawn for the cited reference and citing articles after sorting. CiteSpace, which was developed by Chaomei Chen, is a freely available Java application for visualising and analysing trends and patterns in the scientific literature. The software measures the literature in specific fields to explore the key path and knowledge turning point of discipline evolution. Thereafter, this research draws a series of visual maps, analyses the potential dynamic mechanism of discipline evolution, and explores the frontier of discipline development. The design of the tool is based on the basic assumption that actual scientific knowledge is constantly changing, similar to the gradual obsolescence and emergence of old and new literature, respectively. The so-called scientific domain refers to any boundary range that can be customised at different levels of clustering, including a scientific topic, research field, or discipline. Knowledge domain is represented by scientific literature sample data sources from different fields. This domain can be used to analyse the potential knowledge contained in massive literature data and present its structure, law, and distribution in the form of a knowledge graph.

CiteSpace can effectively help readers acquire an improved understanding of their research fields. It can not show the overall situation of a research field and also highlight some specific important documents in the development process. Specifically, CiteSpace helps answer the following questions: "Which literature is pioneering and iconic in a research field?," "Which literature plays a key role in the development of a research field?," "Which topics occupy the mainstream position in the whole research field?," "How are different research fields related to each other?," and "How did the research frontier based on a certain knowledge base evolve?". CiteSpace provides a new research perspective and a valuable scientific research tool.

This research mainly studies the development status and trend of intelligent media in the recent 10 years by analysing the six dimensions of annual publication quantity, country of publication, institution of publication, author, keywords, and cited references. CiteSpace map has two most direct foundations, namely, (1) disciplinary basis based on citation analysis and (2) technical basis based on information visualisation, thereby intuitively expressing the research results of this study. The current research reveals the main forces, evolution process, research hotspots and topic categories of intelligent media research in the past decade. Through scientific cooperation network, keyword symbiosis network, and keyword clustering analyses, this study reveals the main forces, evolution process, research hotspots, and theme categories of intelligent media research in the recent 10 years. This study uses the results of the knowledge map in predicting the possible development trend of intelligent media in the future.

3.2. Research Object and Data Source. This study selects the core collection of Web of Science (WOS) as a data source. 


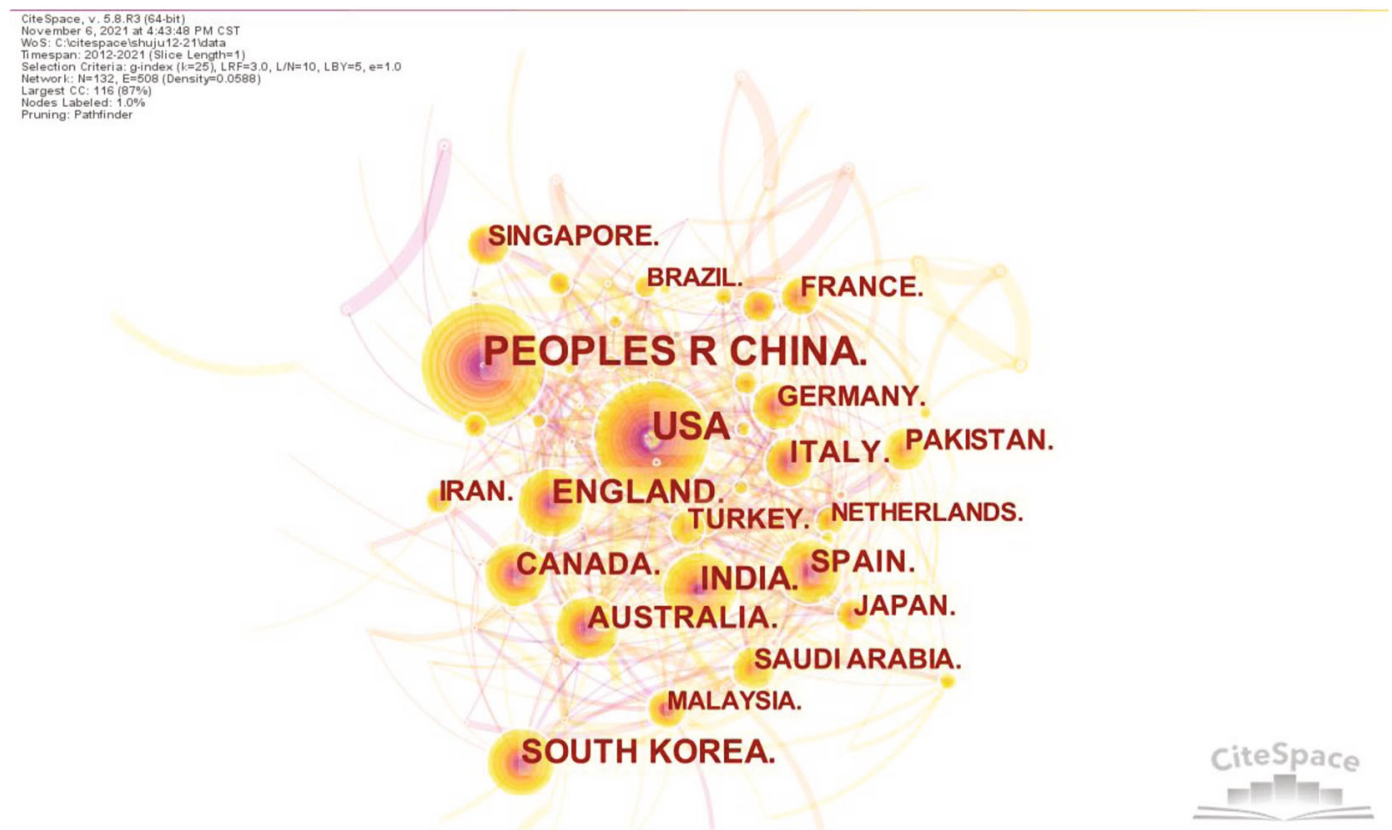

FIgURE 2: Cooccurrence graph of paper publication from a national perspective.

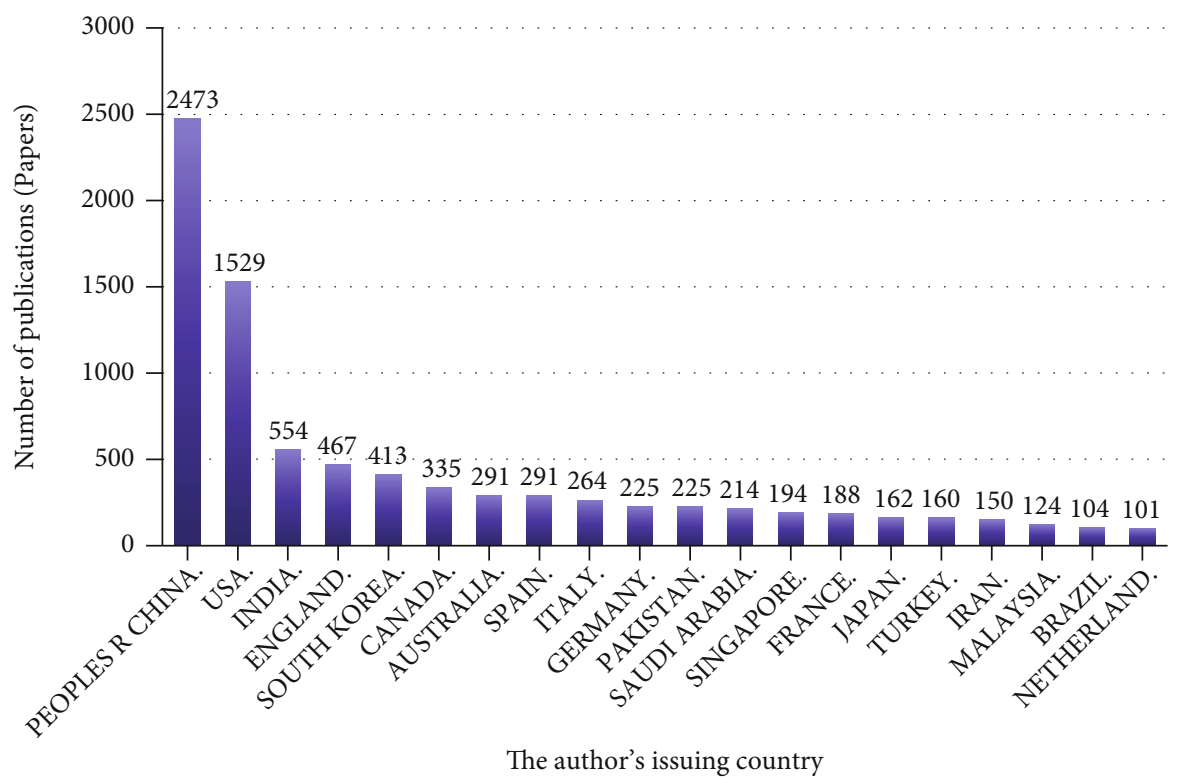

Figure 3: Countries with at least 100 papers.

According to the interpretation and analysis of the research literature in the field of intelligent media mentioned in the literature review, the relevant keywords in intelligent media have been preliminarily determined. This study searches keywords in the WOS database and attempts to unlock the keywords that can reflect the field in the WOS database. "Intelligent media" and "smart media" are used as search words for the trial check, and keywords related to the two fields of "artificial intelligence" and "media" are sorted through trial search. Combined with the interpretation of intelligent media by wireless communications and mobile computing, Li et al. [24] and Qian et al. [25] formulated a retrieval formula for subsequent trial retrieval for the research of key terms in the field of artificial intelligence and common media and enterprises using intelligent technology in social and economic activities. On this basis, the current study repeatedly attempts and adjusts the retrieval formula and browse and compare the retrieval results after each adjustment, excluding the content not related to the subject. Lastly, this study determines the retrieval formula as [TS $=((($ "adaptive system") OR ("machine intelligen $*$ ") OR ("intelligent network") OR ("intelligent systhesis") OR 


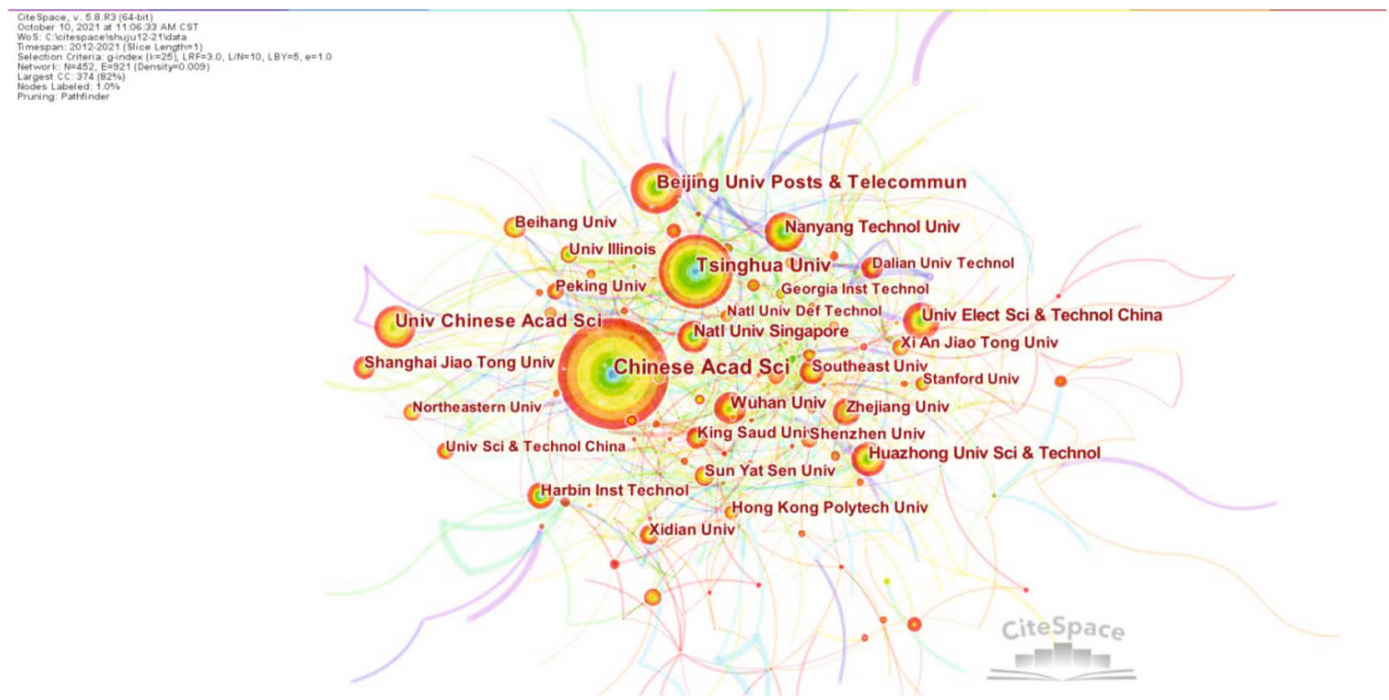

FIgURE 4: Cooccurrence graph of institutions.

("ambient intelligen *") OR ("brain model *") OR ("virtual reality") OR ("augmented reality") OR ("cognitive system") OR ("deep learn *") OR ("algorithm") OR ("expert system") OR ("formal logic *") OR ("heuristic program") OR ("intelligent agent *") OR ("local search *") OR ("prediction model *") OR ("logic program") OR ("reinforcement learning") OR (robot *) OR ("image recogn *") OR ("speech recogn *") OR ("distributed comput *") OR ("system Theor *") OR ("cloud computing") OR ("fuzzy control *") OR ("knowledge engineering") OR ("ubiquitous comput *") OR ("intelligent system") OR ("agent-based model *") OR ("multi-agent system") OR ("neural network") OR ("problem solv *") OR (swarm *) OR ("data proces *") OR ("artificial intelligence") OR ("text minning") OR ("feature recogn *") OR ("pattern analysis") OR ("intelligent creation") OR ("resource allocat *") OR ("immersive experience")) AND $(((($ smart) OR (intell $*))$ AND ((film) OR (television) OR (audio) OR (radio)))) OR ("social media") OR ("mass media") OR ("news media") OR ("short video") OR (Youtube) OR (Blog) OR (facebook) OR (Twitter) OR (QQ) OR (wechat) OR (instagram) OR (TikTok) OR (vimeo) OR (matecafe) OR (dailymotion) OR (liveleak) OR (hulu) OR (viewster) OR (twitch) OR (netflix) OR (vube) OR (weibo) OR (zhihu) OR (bilibili)))]. The data set is limited to "SCIE," time is limited to "2012-2021," language is limited to "English," document types are limited to "article" and "review," retrieval time is October 10,2021, and a total of 7248 records are obtained. Additionally, 145 highly cited papers are obtained through refining for comparison.

\section{Results and Discussion}

4.1. Analysis of Annual Publications. Statistics on annual publication quantity can show the research attention of scholars in intelligent media at different periods. This study used the method of metrological visualisation analysis to calculate the annual number of 7248 retrieved articles (see Figure 1). The result indicated that the number of relevant
TABLE 1: Institutions with at least 40 published papers.

\begin{tabular}{|c|c|c|}
\hline No. & Institution & No. of papers \\
\hline 1 & Chinese Acad Sci & 204 \\
\hline 2 & Tsinghua Univ & 142 \\
\hline 3 & Beijing Univ Posts \& Telecommun & 104 \\
\hline 4 & Univ Chinese Acad Sci & 91 \\
\hline 5 & Nanyang Technol Univ & 85 \\
\hline 6 & Univ Elect Sci \& Technol China & 74 \\
\hline 7 & Huazhong Univ Sci \& Technol & 73 \\
\hline 8 & Natl Univ Singapore & 70 \\
\hline 9 & Wuhan Univ & 67 \\
\hline 10 & Zhejiang Univ & 64 \\
\hline 11 & Harbin Inst Technol & 61 \\
\hline 12 & Shanghai Jiao Tong Univ & 58 \\
\hline 13 & Beihang Univ & 56 \\
\hline 14 & Xidian Univ & 55 \\
\hline 15 & King Saud Univ & 55 \\
\hline 16 & Xi An Jiao Tong Univ & 54 \\
\hline 17 & Southeast Univ & 52 \\
\hline 18 & Univ Illinois & 51 \\
\hline 19 & Shenzhen Univ & 50 \\
\hline 20 & Hong Kong Polytech Univ & 49 \\
\hline 21 & Sun Yat Sen Univ & 48 \\
\hline 22 & Peking Univ & 47 \\
\hline 23 & Dalian Univ Technol & 46 \\
\hline 24 & Northeastern Univ & 46 \\
\hline 25 & Georgia Inst Technol & 45 \\
\hline 26 & Natl Univ Def Technol & 44 \\
\hline 27 & Stanford Univ & 44 \\
\hline 28 & Univ Sci \& Technol China & 43 \\
\hline
\end{tabular}




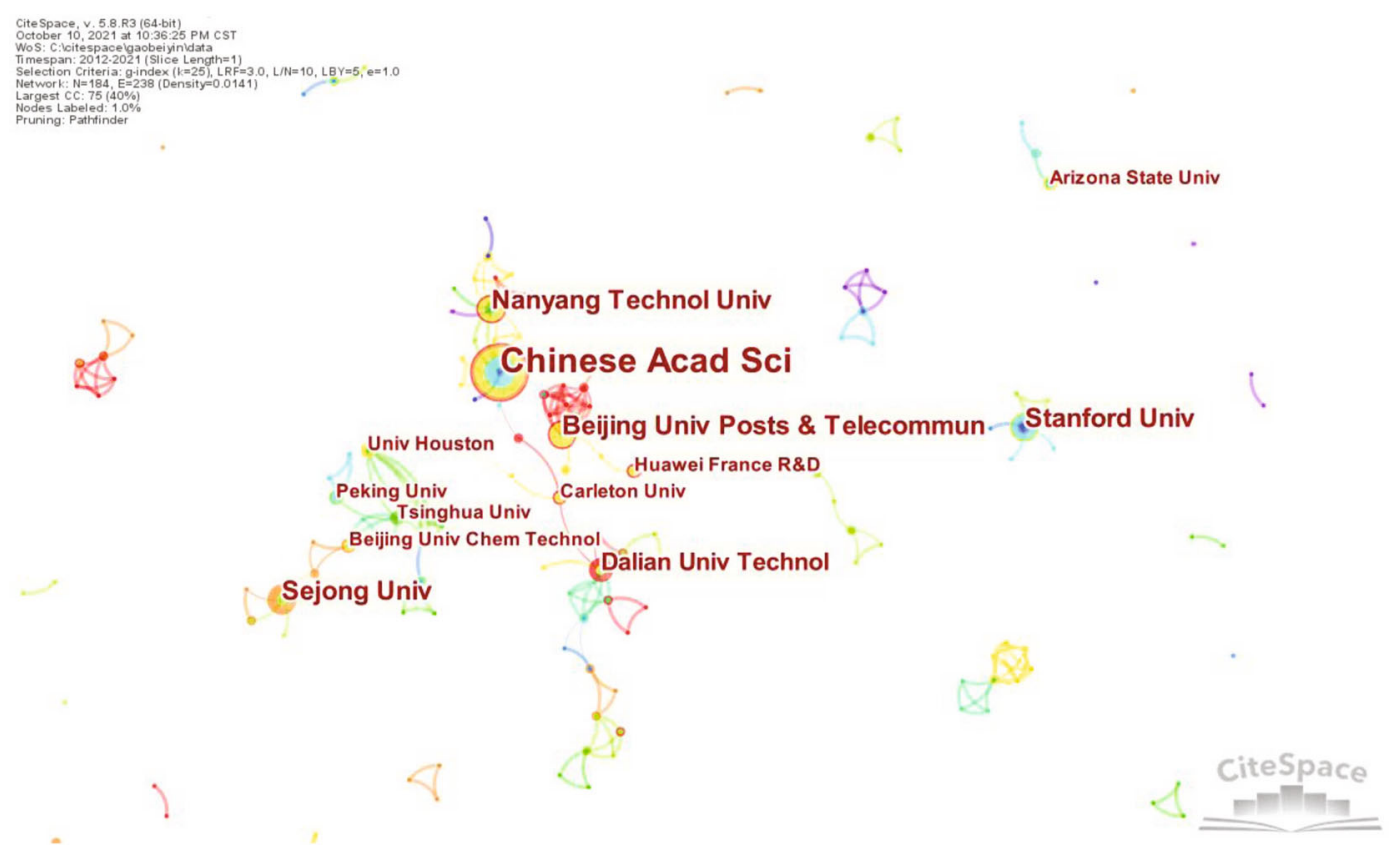

FIgURE 5: Cooccurrence graph of institutions with highly cited literature.

studies on intelligent media by international scholars has been increasing annually. In 2012, there were only 179 articles on the topic of "intelligent media," but the number of articles published in this field reached 596 in 2017, 809 in 2018, 1149 in 2019, and 1787 in 2020. Given that 2021 data are only up to October 10, 2021 (i.e., retrieval time of this study), the number of articles published in this field is expected to exceed 2000 in 2021. Note that since 2017, the annual number of papers published in intelligent media has increased rapidly, and the research heat has not decreased. The general trend is to promote the entry of intelligent technology to the media industry.

4.2. Analysis of the Publication of Paper from a National Perspective. The visual analysis of the author's issuing country by CiteSpace software indicates that the node type is country. Figure 2 shows that countries with over 100 starting documents are obtained. The national cooperation perspective indicates 132 nodes, 508 connections between nodes, and network density of 0.0588 . Note that the current national cooperation in intelligent media research is relatively close. Figure 2 shows that the larger a country's name and the thicker the node ring, the more the total number of articles published by the country. The number of documents issued can also reflect a country's research strength and level in this field. The thicker the connection between nodes, the closer the cooperation between countries.

The People's Republic of China ranks first in the number of published papers, with 2473 papers. The United States of America (the USA) and India are second and third with 1529 and 554 articles, respectively (see Figure 3). Note that the People's Republic of China and the USA have high proportions of documents in this field. Presently, various countries are closely connected in intelligent media research.
4.3. Analysis of Productive Institutions. The visual analysis of the document issuing organisation by CiteSpace software indicates that the node type is organisation. Organisations with over 40 published papers are obtained (see Figure 4). The cooccurrence graph of institutional cooperation shows 452 nodes, 921 connections between nodes, and network density of 0.009 . Note that there is cooperation amongst institutions in intelligent media research, but such cooperation is insufficient. Figure 4 shows that the larger the name of organisations and the thicker the annual rings of the nodes representing the total number of documents issued by organisations reflect the research strength and level of these organisations in this field. The thicker the connection between nodes, the closer the cooperation between representative organisations.

The top three institutions are Chinese Academy of Sciences (CAS), Tsinghua University, and Beijing University of Posts and Telecommunications. A total of 28 institutions have a volume of at least 40 articles, 21 of which are Chinese universities and scientific research institutions and 4 are American universities (see Table 1). This result is consistent with the cooccurrence graph of the national document volume in the second part. Note that China's document volume is dominant in this field, and the cooperation between domestic institutions is relatively close.

A total of 145 highly cited documents obtained from refining were visually analysed based on CiteSpace software (see Figure 5). Amongst the highly cited documents, CAS has the most number of documents and has close cooperation with major universities and institutions locally and overseas.

CAS has the most number of papers, with a total of 12 . There are 13 institutions with at least 3 articles, of which 7 are Chinese universities and scientific research institutions 
TABLE 2: Institutions that have published at least three highly cited papers.

\begin{tabular}{lcc}
\hline No. & Institution & No. of papers \\
\hline 1 & Chinese Acad Sci & 12 \\
2 & Stanford Univ & 6 \\
3 & Sejong Univ & 6 \\
4 & Beijing Univ Posts \& Telecommun & 6 \\
5 & Nanyang Technol Univ & 6 \\
6 & Dalian Univ Technol & 5 \\
7 & Peking Univ & 3 \\
8 & Arizona State Univ & 3 \\
9 & Huawei France R\&D & 3 \\
10 & Univ Houston & 3 \\
11 & Carleton Univ & 3 \\
12 & Tsinghua Univ & 3 \\
13 & Beijing Univ Chem Technol & 3 \\
\hline
\end{tabular}

and 3 are American universities (see Table 2). Figure 5 and Table 2 show that CAS ranks first in intelligent media in terms of the number of publications and number of highly cited literature. The overall proportion of Chinese universities is also high. This result shows that China's research in intelligent media is at the forefront and has achieved good results in terms of quantity and quality.

4.4. Author Analysis. The author's cooccurrence analysis can reflect the cooperative relationship between the core authors and researchers in this field. Price law points out that the author's calculation formula is as follows:

$$
N_{\min }=0.749 \sqrt{N_{\max }}
$$

where $N_{\max }$ is the number of articles published by the author with the largest number of articles in a certain field. If the number of articles published by an author is not below $N_{\text {min }}$, then the author is called a high-yield author. Table 3 shows that if the value $N_{\max }$ is 21 , then $N_{\min } \approx 3.432$ is obtained and the integer is 4 . Therefore, if there are at least 4 published papers, then the author is called a high-yield author.

According to the price law, only when the high-yield author group accounts for $50 \%$ of the total can the discipline high-yield author group be formed. After calculation, the number of high-yield authors in this field accounts for only $7.19 \%$ of the total number of papers, indicating that researchers in intelligent media are relatively scattered and have not formed a high-yield author group. Table 3 shows that the author with the most number of articles (21) is Wei Wang of Sichuan University, followed by Yingchang Liang (with 14) of the University of Electronic Science and Technology. Note that the high-yield authors began to publish more papers in intelligent media in 2016.

The graph shows 529 nodes, 422 connections between nodes, and network density of 0.003 . Cooperation of
TABLE 3: High-yield authors with at least six papers.

\begin{tabular}{|c|c|c|c|}
\hline No. & Author & No. of papers & Start year \\
\hline 1 & Wei Wang & 21 & 2015 \\
\hline 2 & Yingchang Liang & 14 & 2019 \\
\hline 3 & Akshi Kumar & 12 & 2019 \\
\hline 4 & Jun Zhang & 12 & 2017 \\
\hline 5 & Jian Wang & 12 & 2019 \\
\hline 6 & Erik Cambria & 11 & 2015 \\
\hline 7 & Mugen Peng & 10 & 2019 \\
\hline 8 & Alagan Anpalagan & 9 & 2013 \\
\hline 9 & Muhammad Imran & 9 & 2020 \\
\hline 10 & Wei Huang & 9 & 2018 \\
\hline 11 & shiqiang yang & 8 & 2013 \\
\hline 12 & Lei Zhang & 7 & 2019 \\
\hline 13 & Fadi Alturjman & 7 & 2020 \\
\hline 14 & Koklim Alvin Yau & 7 & 2014 \\
\hline 15 & Hua Wang & 7 & 2017 \\
\hline 16 & M Shamim Hossain & 7 & 2018 \\
\hline 17 & Tatseng Chua & 7 & 2014 \\
\hline 18 & Min Chen & 7 & 2014 \\
\hline 19 & Junaid Qadir & 7 & 2016 \\
\hline 20 & Chang Liu & 7 & 2019 \\
\hline 21 & Jing Liu & 6 & 2014 \\
\hline 22 & Arun Kumar Sangaiah & 6 & 2018 \\
\hline 23 & Liang Xiao & 6 & 2015 \\
\hline 24 & Hui Li & 6 & 2020 \\
\hline 25 & Bin Zhang & 6 & 2017 \\
\hline 26 & Chao Wang & 6 & 2013 \\
\hline 27 & Ting Liu & 6 & 2016 \\
\hline 28 & Ming Li & 6 & 2020 \\
\hline 29 & Qihui Wu & 6 & 2013 \\
\hline 30 & Hongfei Lin & 6 & 2019 \\
\hline 31 & Khan Muhammad & 6 & 2020 \\
\hline
\end{tabular}

researchers in this field is not close (see Figure 6). Particularly, teams with relatively more papers and outstanding cooperation have two cooperative relationships (1) Jun Zhang and Qingqing Wu and Ming $\mathrm{Li}$, amongst others and (2) Yingchang Liang and Muhammad Ali-Imran and Lei Zhang, amongst others.

\subsection{High-Frequency Keyword Analysis}

4.5.1. Cooccurrence Analysis of High-Frequency Keywords. Keywords are highly generalised to the literature theme. Keyword analysis of the literature is aimed at revealing the literature theme, which can reflect the research hotspot, research direction, internal relationship, and importance of this research field.

CiteSpace software was used in conducting cooccurrence analysis of the keywords of 7248 retrieved literature and removing words irrelevant to the research field (see Figure 7). Particularly, there are 526 nodes 689 connections 


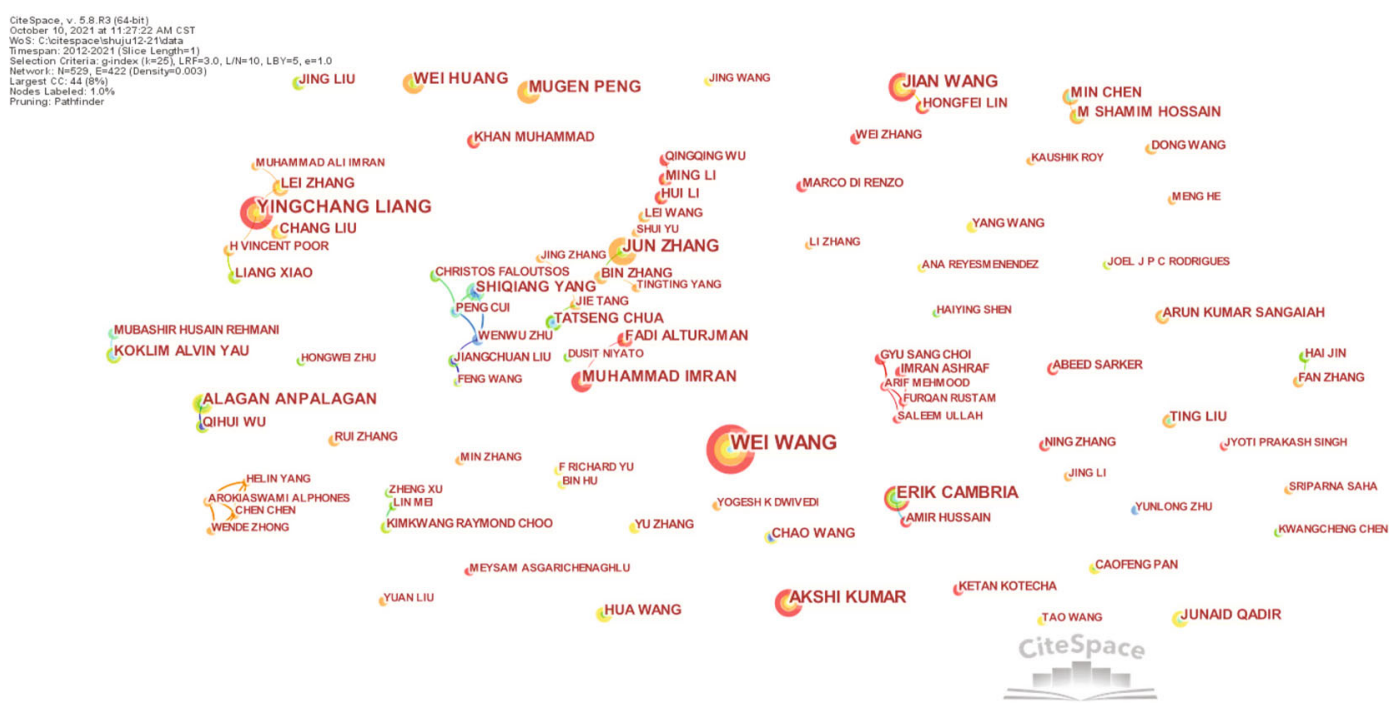

Figure 6: Cooccurrence graph of high-yield authors.

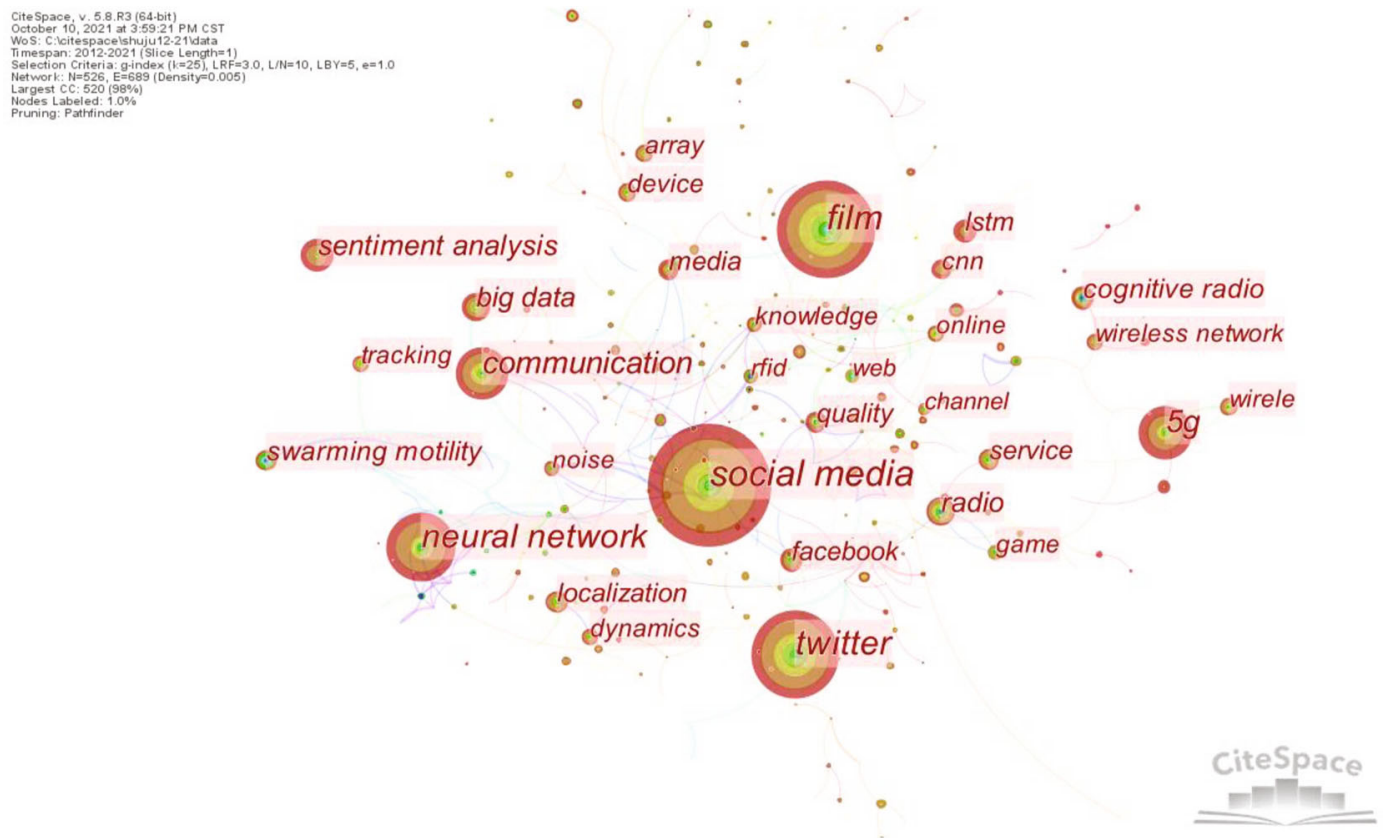

Figure 7: Cooccurrence graph of keywords.

between nodes and network density is 0.005 . The larger the node and font size the higher the keyword usage frequency. The thicker the connection the closer the connection between the two keywords. The greater the centrality the more important the keywords. The general belief is that keywords with centrality above 0.1 are important and keywords with high frequency and great centrality can be used as research hotspots.

Keywords with frequency of over 40 are analysed (see Table 4). The frequency and centrality of film and communication are very high, indicating that the two keywords are more important. Related keywords include social media, twitter, neural network, 5G, sentient analysis, radio, and big data.
4.5.2. Keyword Cluster Analysis. Keyword clustering analysis can reflect the most popular research topic in this field.

CiteSpace conducts cluster analysis on high-frequency keywords in intelligent media. It sets the cluster number to 6 and obtains 6 clustering points about intelligent media (see Figure 8): film, social media, machine learning, swarming motility, data mining, and artificial intelligence. Each cluster is composed of closely related keywords. When the cluster $S$ value is above 0.5 , clustering is reasonable. When the clustering $S$ value is above 0.7 , clustering results are convincing. Given that the $S$ values of the six clusters shown in Figure 8 are above 0.7 , the clustering results of this time have a high degree of credibility. 
TABLE 4: High-frequency keywords and their centrality list.

\begin{tabular}{|c|c|c|c|c|}
\hline No. & Keyword & Frequency & Centrality & Start year \\
\hline 1 & Social media & 252 & 0.02 & 2013 \\
\hline 2 & Film & 200 & 0.23 & 2014 \\
\hline 3 & Twitter & 180 & 0.02 & 2015 \\
\hline 4 & Neural network & 145 & 0.07 & 2013 \\
\hline 5 & Communication & 115 & 0.21 & 2012 \\
\hline 6 & $5 \mathrm{G}$ & 112 & 0.03 & 2015 \\
\hline 7 & Sentiment analysis & 70 & 0.01 & 2017 \\
\hline 8 & Radio & 67 & 0.02 & 2012 \\
\hline 9 & Big data & 64 & 0.01 & 2016 \\
\hline 10 & Cognitive radio & 57 & 0.02 & 2012 \\
\hline 11 & Swarming motility & 54 & 0.02 & 2012 \\
\hline 12 & Time & 52 & 0.02 & 2015 \\
\hline 13 & Service & 52 & 0.09 & 2013 \\
\hline 14 & Facebook & 51 & 0.08 & 2014 \\
\hline 15 & Quality & 50 & 0.07 & 2012 \\
\hline 16 & LSTM & 49 & 0 & 2019 \\
\hline 17 & Localization & 49 & 0.03 & 2013 \\
\hline 18 & Media & 48 & 0.03 & 2014 \\
\hline 19 & Device & 45 & 0.07 & 2015 \\
\hline 20 & Array & 43 & 0.05 & 2015 \\
\hline 21 & $\mathrm{CNN}$ & 42 & 0.01 & 2019 \\
\hline 22 & Dynamics & 42 & 0.05 & 2013 \\
\hline 23 & Knowledge & 41 & 0.04 & 2012 \\
\hline
\end{tabular}

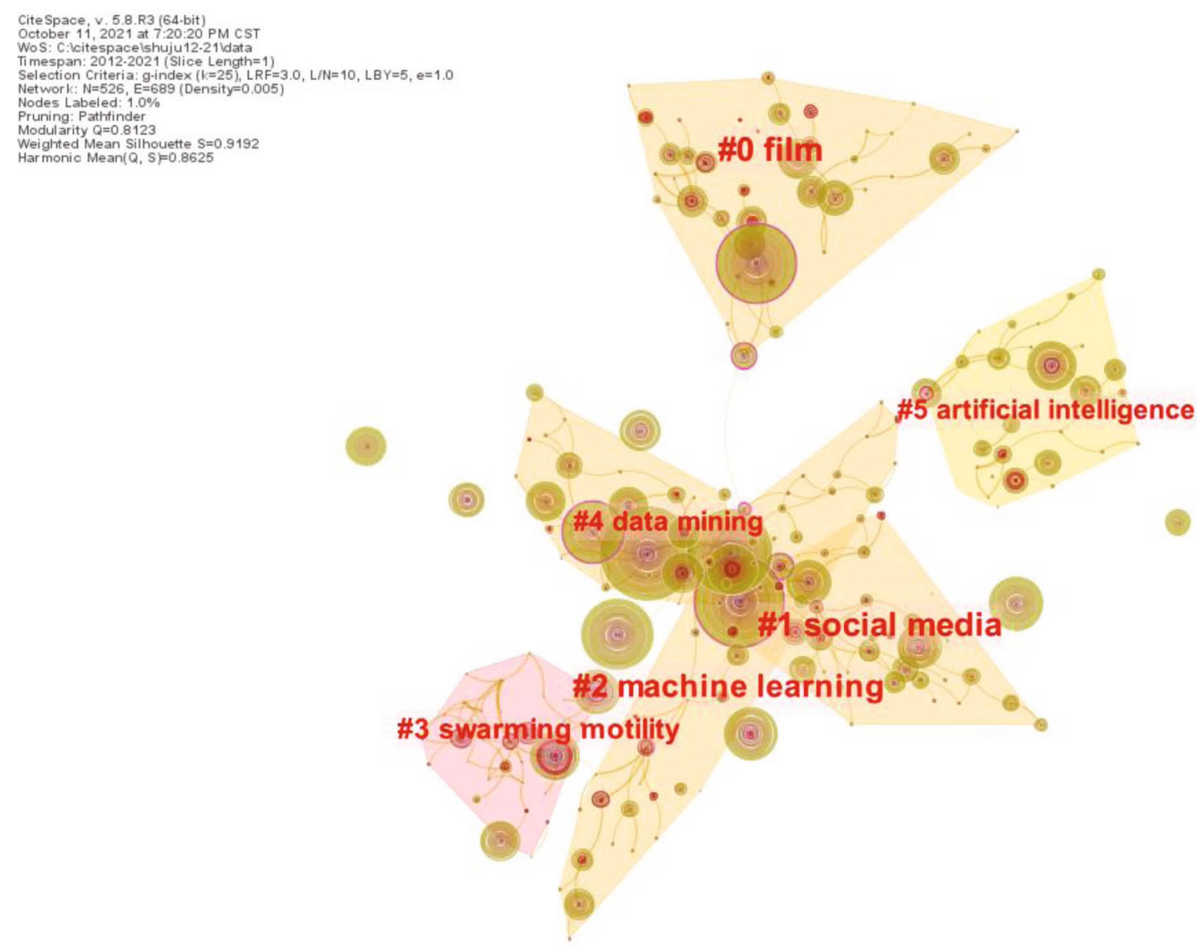

FIgURE 8: Keyword cluster analysis graph. 


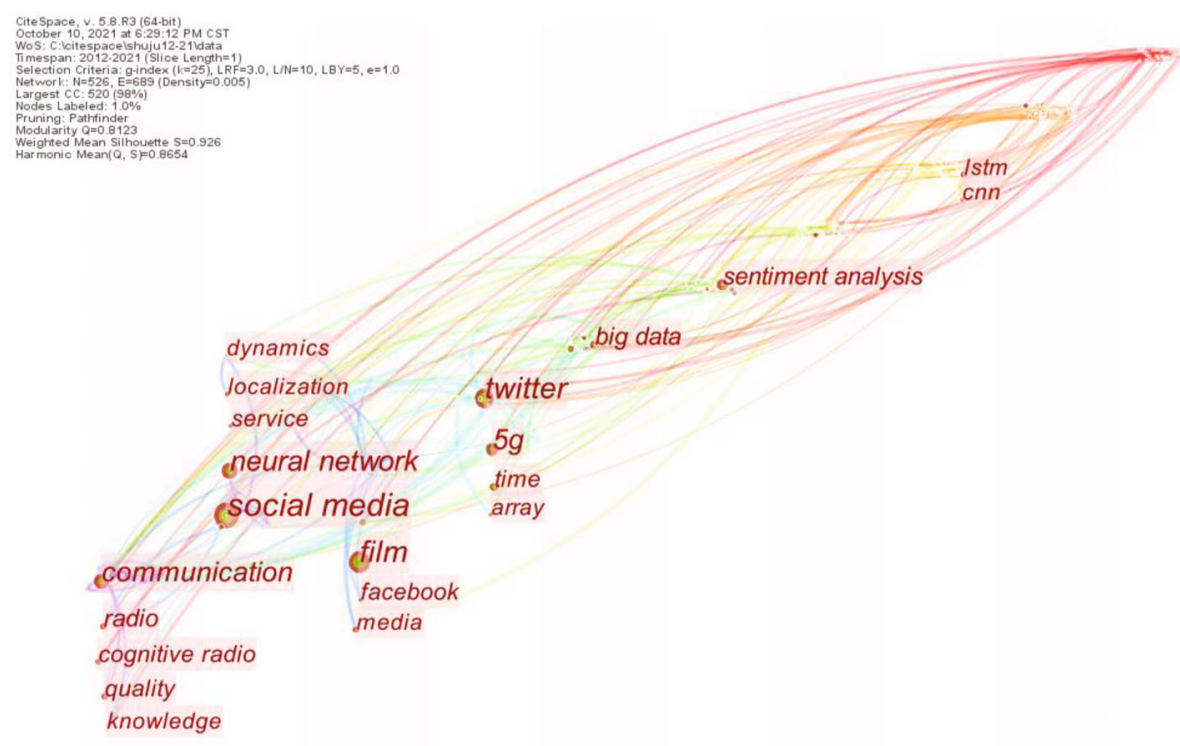

Figure 9: Keyword time zone graph.

\begin{tabular}{lccccc}
\multicolumn{1}{c}{ Keywords } & Year & Strength & Begin & End & 2012-2021 \\
Swarming motility & 2012 & 13.25 & 2012 & 2017 & \\
Cognitive radio & 2012 & 7.3 & 2012 & 2015 & \\
Rfid & 2012 & 6.45 & 2012 & 2014 & \\
Context & 2012 & 4.72 & 2012 & 2014 & \\
Intelligibility & 2012 & 3.13 & 2012 & 2015 & \\
Radio & 2012 & 2.49 & 2012 & 2014 & \\
Genetic algorithm & 2012 & 3.47 & 2013 & 2015 & \\
Game & 2012 & 3.27 & 2013 & 2018 & \\
Video & 2012 & 2.69 & 2013 & 2015 & \\
Web & 2012 & 8.49 & 2014 & 2016 & \\
Time & 2012 & 6.04 & 2015 & 2017 & \\
Complex network & 2012 & 4.72 & 2016 & 2017 & \\
Fiber & 2012 & 4.18 & 2017 & 2019 & \\
Cognitive radio network & 2012 & 3.16 & 2017 & 2018 & - \\
Mobile & 2012 & 3.4 & 2019 & 2021 & \\
& & & & & \\
& & & &
\end{tabular}

FIGURE 10: Ranking of mutation keywords.

4.5.3. Keyword Time Zone Analysis. CiteSpace software analyses the time zone distribution of high-frequency keywords (see Figure 9). The topic path map can reflect the cooccurrence relationship of keywords in a specific time range and the development context and research trend of a certain field. Figure 9 shows that the location of each circle represents the earliest publication year of an article in which the keyword is located, the thickness of the growth ring represents the heat of the research, the line between keywords represents the cooccurrence relationship, and the trend of the line represents the development of this research direction. Figure 9 shows a trend in the development of highfrequency keywords in intelligent media in the past decade from 2012 to 2021. The keyword with high frequency in 2013 is social media, which has continued to date.

4.5.4. Keyword Burst Analysis. Emergent words are key terms in which keywords suddenly increase or their use frequency has increased significantly in a certain period. Mutation words can show some cutting-edge research fields of intelligent media research. Figure 10 shows that a total of 15 mutation words are obtained. Amongst them, the strongest is swarming mobility, with mutation intensity of 13.25. The first year of its emergence was 2012 and ended in 2017. Swarming motility has a longer time of existence, which was also the area of interest in the early years and 
TABLE 5: Citation frequency and starting year of highly cited references.

\begin{tabular}{|c|c|c|c|}
\hline No. & Paper & Frequency & $\begin{array}{l}\text { Start } \\
\text { year }\end{array}$ \\
\hline 1 & LeCun Y, 2015, NATURE, V521, P436, DOI 10.1038/nature14539 & 143 & 2015 \\
\hline 2 & Devlin J, 2019, P NAACL HLT, V0, P0, DOI 10.18653/v1/N19-1423 & 120 & 2019 \\
\hline 3 & Kingma DP, 2017, ICLR, V0, P0 & 115 & 2017 \\
\hline 4 & $\begin{array}{c}\text { Kaiming He, 2016, } 2016 \text { IEEE Conference on Computer Vision and Pattern Recognition (CVPR), V0, P770, } \\
\text { DOI 10.1109/CVPR.2016.90 }\end{array}$ & 92 & 2016 \\
\hline 5 & Krizhevsky A, 2017, COMMUN ACM, V60, P84, DOI 10.1145/3065386 & 81 & 2017 \\
\hline 6 & Goodfellow I, 2016, ADAPT COMPUT MACH LE, V0, P1 & 60 & 2016 \\
\hline 7 & He KM, 2016, PROC CVPR IEEE, V0, P770, DOI 10.1109/CVPR.2016.90 & 57 & 2016 \\
\hline 8 & Cambria E, 2016, IEEE INTELL SYST, V31, P102, DOI 10.1109/MIS.2016.31 & 56 & 2016 \\
\hline 9 & Pennington J, 2014, P EMNLP C, V0, P1532, DOI DOI 10.3115/V1/D14-1162 & 54 & 2014 \\
\hline 10 & Bojanowski P, 2017, T ASSOC COMPUT LING, V5, P135 & 53 & 2017 \\
\hline 11 & Russakovsky O, 2015, INT J COMPUT VISION, V115, P211, DOI 10.1007/s11263-015-0816-y & 46 & 2015 \\
\hline 12 & Vosoughi S, 2018, SCIENCE, V359, P1146, DOI 10.1126/science.aap9559 & 45 & 2018 \\
\hline 13 & Simonyan K, 2014, ARXIV14091556, V0, P0, DOI 10.1109/CVPR.2015.7298594] & 45 & 2014 \\
\hline 14 & Mnih V, 2015, NATURE, V518, P529, DOI 10.1038/nature14236 & 45 & 2015 \\
\hline 15 & Schmidhuber J, 2015, NEURAL NETWORKS, V61, P85, DOI 10.1016/j.neunet.2014.09.003 & 43 & 2015 \\
\hline 16 & Kai Shu, 2017, ACM SIGKDD Explorations Newsletter, V19, P22, DOI 10.1145/3137597.3137600 & 42 & 2017 \\
\hline 17 & He K, 2016, P IEEE C COMPUTER VI, V0, P770, DOI DOI 10.1109/CVPR.2016.90 & 42 & 2016 \\
\hline 18 & Ma J, 2016, P 25 INT JOINT C ART, V0, P3818 & 41 & 2016 \\
\hline 19 & Wu QQ, 2019, IEEE T WIREL COMMUN, V18, P5394, DOI 10.1109/TWC.2019.2936025 & 40 & 2019 \\
\hline
\end{tabular}

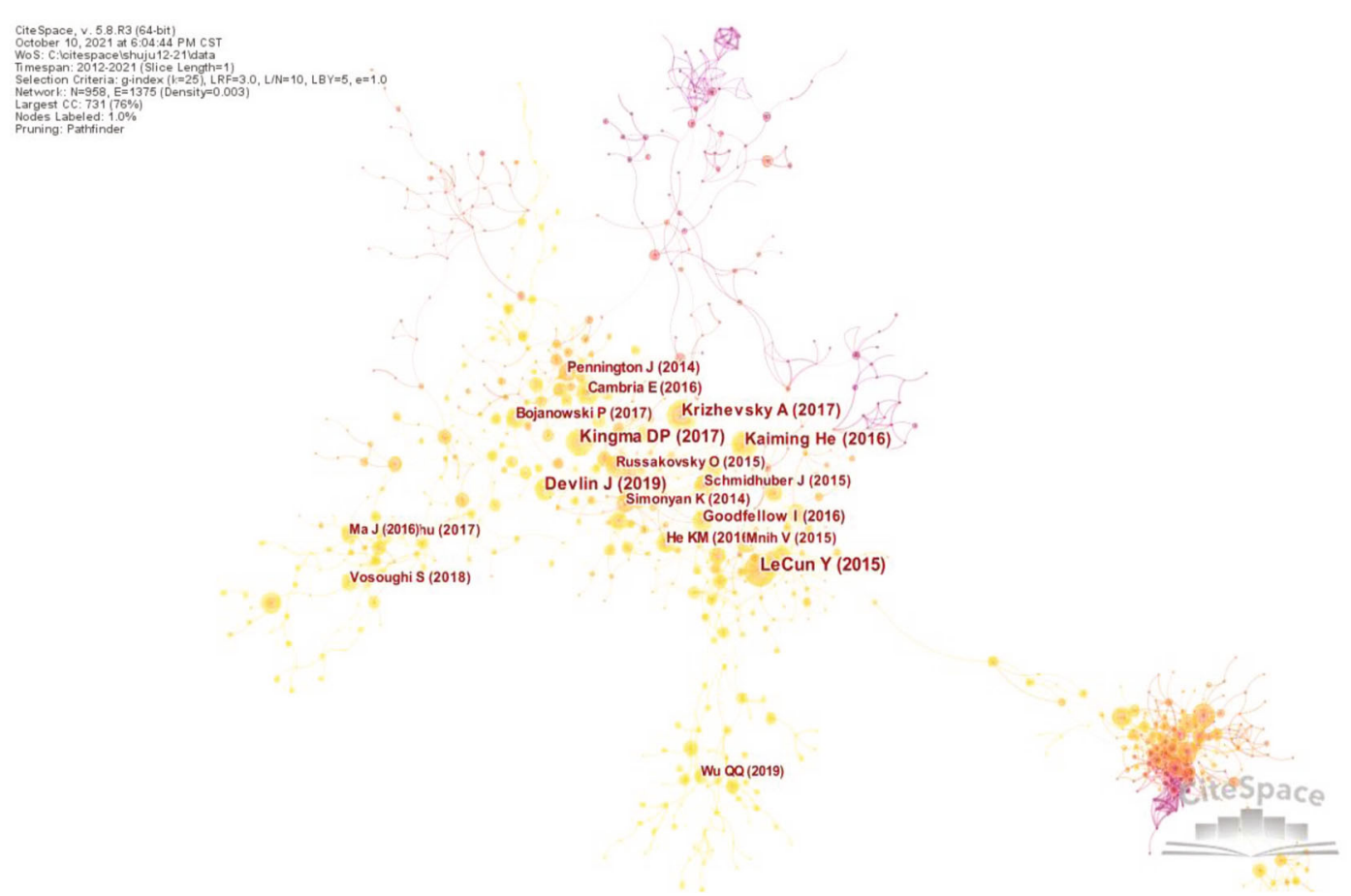

Figure 11: Cooccurrence graph of highly cited documents (citation frequency of at least 40).

lasted a long time. With the advent of the $5 \mathrm{G}$ era, mobile has been a theme of recent interest since 2019 .

4.6. Document Cocitation Analysis. Cocitation analysis of references primarily is aimed at studying key documents and research hotspots in this field. Cocitation clustering reflects the popular topics and research directions of common concern amongst clustered documents. The cocitation time zone graph can reflect the evolution process of this field in the time dimension. 


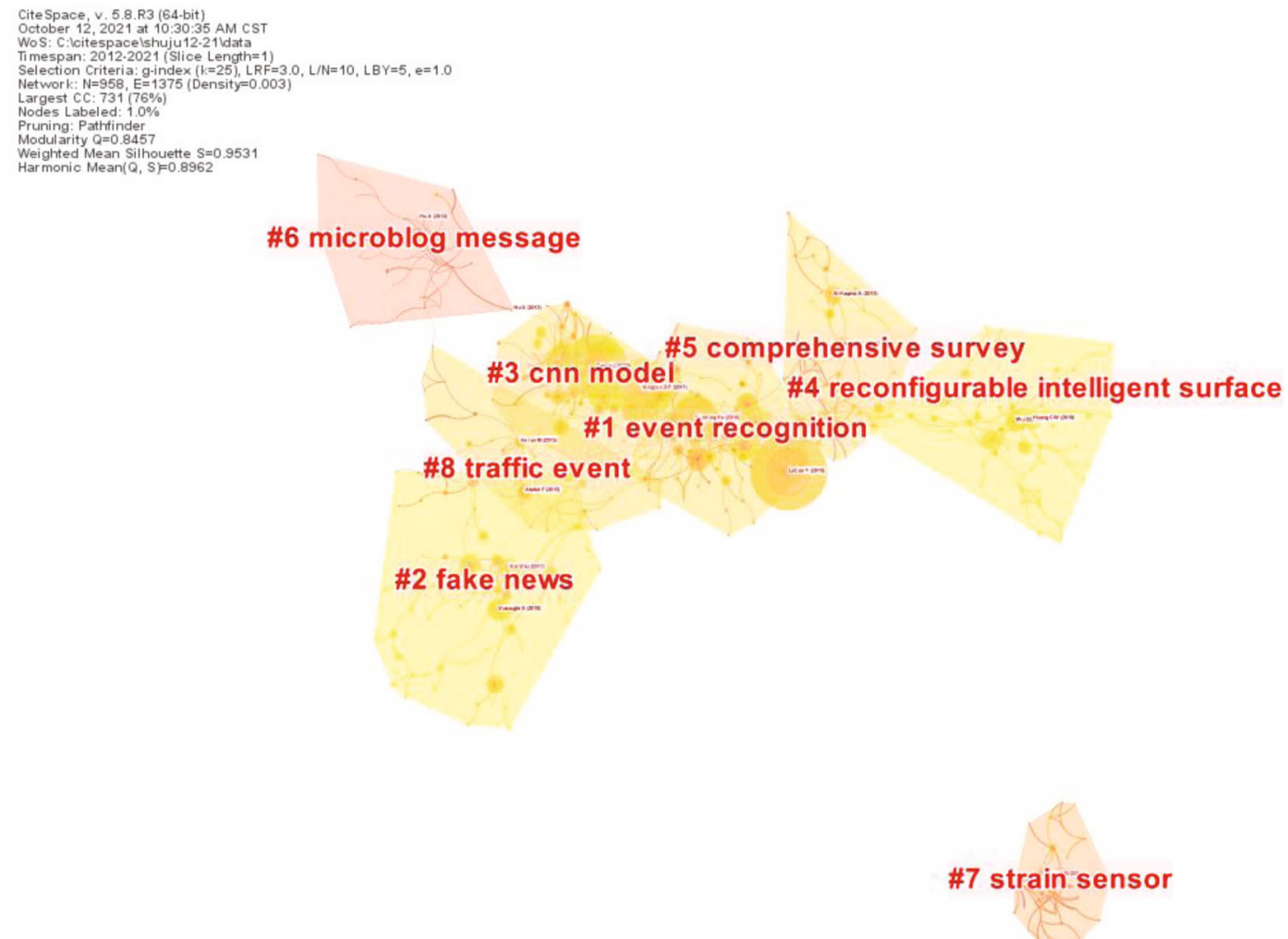

FIGURE 12: Clustering graph of highly cited documents.
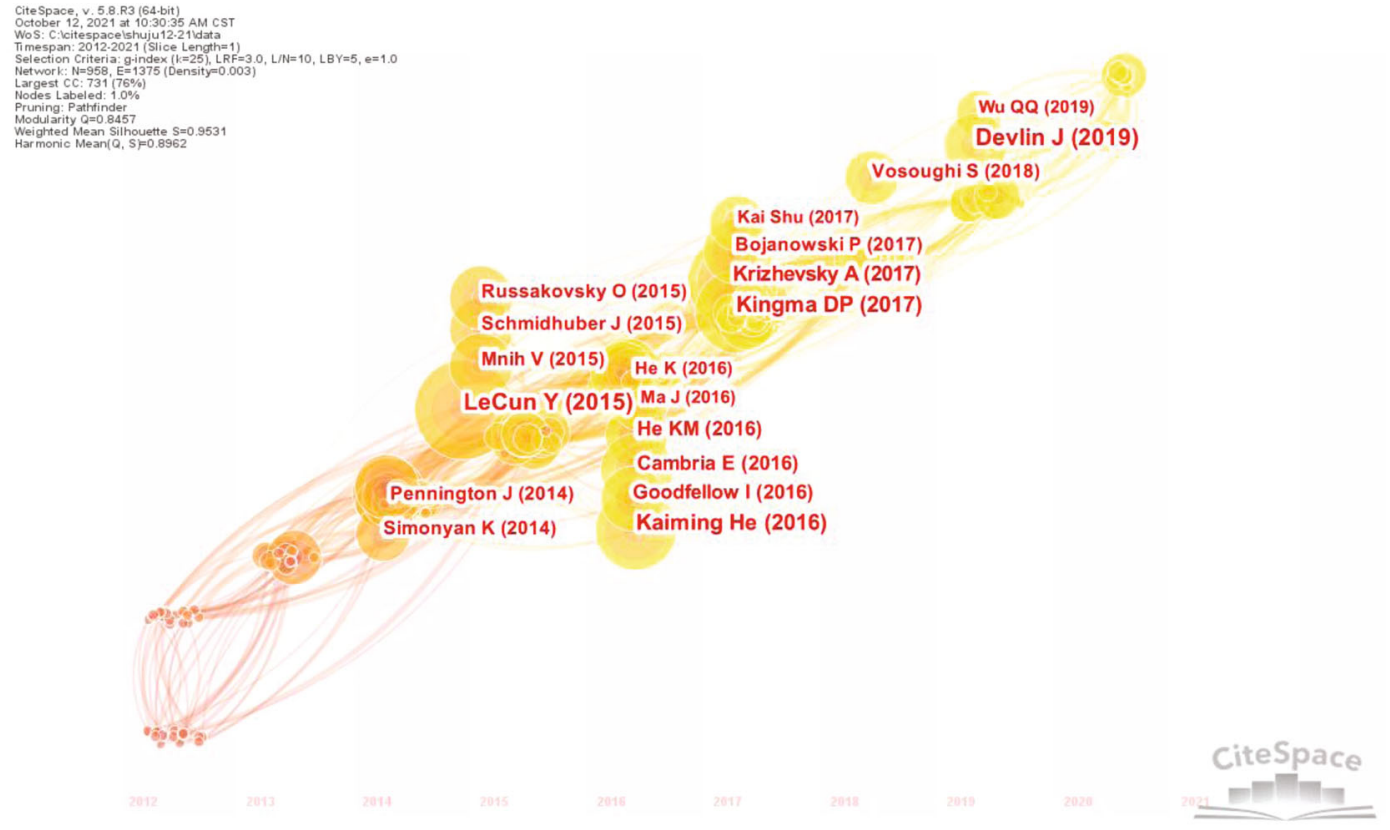

FIgURE 13: Time zone graph of highly cited documents.

CiteSpace conducted cooccurrence analysis and cluster analysis on literature cocitation (see Table 5 and Figure 11). References with citation frequency of at least 40 are concentrated in 2015-2016 from the time of publication, which is relatively early. In terms of content, it is mainly aimed at artistic intelligence, news, Cable News Network (CNN), and other fields. These cited references lay the foundation for the subsequent study of intelligent media.

Cluster analysis was conducted on the cited literature. The number of clusters in this cluster was set to 9 , of which 
the No. 0 cluster point was electronic skin, which is inconsistent with the theme of "intelligent media" studied in this research. Therefore, it was removed. Lastly, eight cluster points on intelligent media references were obtained. Subject words of the main research directions in the recent 10 years are event recognition, fake news, CNN model, reconfigurable intelligent surface, comprehensive survey, microblog message, strain sensor, and traffic event (see Figure 12).

According to CiteSpace, highly cited literature was analysed. If there is less literature in a time zone (see Figure 13), then there are less influential achievements in that time zone. By contrast, if there is more literature in a time zone, then numerous influential achievements have accumulated in that time zone. The connection of nodes between time zones indicates the inheritance of the research. Figure 13 shows that in the past 10 years, there were numerous literature in the three-year time zone from 2015 to 2017, which accumulated many influential achievements in this field and laid a certain research foundation for the subsequent research in intelligent media. Given that highly cited literature also involves time delay, papers in recent years have not formed a highly cited effect. With the passage of time, the results accumulated in recent years will continue for follow-up research, and high-quality papers will also be highly cited.

\section{Conclusion}

This research conducted a bibliometric analysis of SCIE retrieved journals from 2012 to 2021 and provided beneficial insights into document types, distribution of countries, regions and institutions, geographical distribution of authors, co-author network, and content analysis. The following conclusions can be drawn according to the development and analysis of research:

(1) In the past decade, the number of papers in intelligent media has been increasing annually. Since 2017, the speed has accelerated. From the perspective of countries that issue papers, the People's Republic of China and the USA have high proportions of documents in this field. Presently, the research of various countries in intelligent media is closely connected

(2) From the perspective of institutions that issue papers, CAS has published the most number of papers. However, in intelligent media research, cooperation amongst institutions is not close because intelligent media is a research topic that has only emerged in recent years. From the perspective of authors, researchers in intelligent media are relatively scattered and have yet to form a highyielding discipline writer group

(3) In intelligent media research, a high-frequency word in the last decade is social media, which shows that intelligent media is closely related to social media. Sharing of social media is essential to meet social needs but not necessarily the needs of the target object. Intelligent media uses algorithm push, tap the needs of the audience, and push the information that meets the needs. Social media will eventually evolve into intelligent media

(4) Judging from the increasing number of papers annually, the general trend is to promote the entry of intelligent technology to the media industry. The key is how to integrate with the existing media business forms or develop new media forms and platforms. According to the law of technological innovation, the best organisational form conducive to the promotion of innovative business is to establish new institutions, adopt new technologies and operate new businesses

(5) Presently, the focus should be research on social issues caused by intelligent media. The privacy, addiction, and risk caused by intelligent media are also important factors affecting the development of intelligent media. Only by studying in advance and preparing scientifically can we prevent problems before they happen

The research on intelligent media presents an interdisciplinary trend. The academic field of intelligent media comprises computer and communication scholars, as well as numerous scholars from communication, journalism, management, and philosophy. With the help of their professional knowledge and research perspective, they discuss the operation mechanism of algorithms, legal supervision, ethical risks and other issues. This aspect shows that systematic problems brought by intelligent media to information communication and even the entire society go beyond the scope of a single discipline and require the joint participation of multiple disciplines.

\section{Data Availability}

The data used to support the findings of this study are available from the corresponding author upon request.

\section{Conflicts of Interest}

The authors declare no conflicts of interest.

\section{Acknowledgments}

We thank Steven Pagel of Philadelphia, USA, for his help in writing this paper. This research was funded by the National Social Science Fund Art Project (17BC059).

\section{References}

[1] M. E. Pollack, "Intelligent technology for an aging population: the use of AI to assist elders with cognitive impairment," $A I$ Magazine, vol. 26, no. 2, pp. 9-24, 2005.

[2] C. Brooks, J. Greer, and C. Gutwin, "The data-assisted approach to building intelligent technology-enhanced learning environments," in Learning analytics, pp. 123-156, Springer, New York, NY, 2014. 
[3] N. Marres, "What if nothing happens? Street trials of intelligent cars as experiments in participation," in TechnoScienceSociety: Technological Reconfigurations of Science and Society, vol. 30, pp. 111-130, Springer, Cham, 2020.

[4] C. Jin, T. Wang, X. Li et al., "A transformer generative adversarial network for multi-track music generation," CAAI Transactions on Intelligence Technology, vol. 2021, pp. 1-12, 2021.

[5] J. Zhang, S. O. Williams, and H. Wang, "Intelligent computing system based on pattern recognition and data mining algorithms," Sustainable Computing: Informatics and Systems, vol. 20, pp. 192-202, 2018.

[6] Y. Liu, R. Bao, J. Tao, J. Li, M. Dong, and C. Pan, "Recent progress in tactile sensors and their applications in intelligent systems," Science Bulletin, vol. 65, no. 1, pp. 70-88, 2020.

[7] G. J. Cheng, L. T. Liu, X. J. Qiang, and Y. Liu, "Industry 4.0 development and application of intelligent manufacturing," in International Conference on Information System and Artificial Intelligence (ISAI), pp. 407-410, Hong Kong, China, 2016.

[8] Y. Xie, Y. Yin, W. Xue, H. Shi, and D. Chong, "Intelligent supply chain performance measurement in Industry 4.0," Systems Research and Behavioral Science, vol. 37, no. 4, pp. 711-718, 2020.

[9] H. C. Sang, S. Kang, and Y. J. Jeon, "Personalized recommendation system based on product specification values," Expert Systems with Applications, vol. 31, no. 3, pp. 607-616, 2006.

[10] Z. Cui, X. Xu, F. Xue et al., "Personalized recommendation system based on collaborative filtering for IoT scenarios," IEEE Transactions on Services Computing, vol. 13, no. 4, pp. 685$695,2020$.

[11] Y. Tian, B. Zheng, Y. Wang, Y. Zhang, and Q. Wu, "College library personalized recommendation system based on hybrid recommendation algorithm," in Procedia CIRP, vol. 83, pp. 490-494, Elsevier, 2019.

[12] M. Yan, H. Yuan, Z. Li, Q. Lin, and J. Li, "Energy savings of wireless communication networks based on mobile user environmental prediction," Journal of Environmental Protection and Ecology, vol. 22, no. 1, pp. 206-217, 2021.

[13] N. Thurman, J. Moeller, N. Helberger, and D. Trilling, My friends, editors, algorithms, and I: examining audience attitudes to news selection, vol. 7, no. 4, 2019Social Science Electronic Publishing, 2019.

[14] P. Lenhart and D. Herzog, Combining content-based and collaborative filtering for personalized sports news recommendations, CBRecSys@ RecSys, 2016.

[15] J. Möller, D. Trilling, N. Helberger, and B. van Es, "Do not blame it on the algorithm: an empirical assessment of multiple recommender systems and their impact on content diversity," Information, Communication \& Society, vol. 21, no. 7, pp. 959977, 2018.

[16] J. Huenteler, T. S. Schmidt, J. Ossenbrink, and V. H. Hoffmann, "Technology life-cycles in the energy sector - technological characteristics and the role of deployment for innovation," Technological Forecasting and Social Change, vol. 104, pp. 102-121, 2016.

[17] N. Diakopoulos, “Algorithmic accountability," Digital Journalism, vol. 3, no. 3, pp. 398-415, 2015.

[18] A. Hanelt, R. Bohnsack, D. Marz, and C. Antunes Marante, "A systematic review of the literature on digital transformation: insights and implications for strategy and organizational change," Journal of Management Studies, vol. 58, no. 5, pp. 1159-1197, 2021.
[19] S. Vosoughi, D. Roy, and S. Aral, "The spread of true and false news online," Science, vol. 359, no. 6380, pp. 1146-1151, 2018.

[20] C. J. Vargo, L. Guo, and M. A. Amazeen, "The agenda-setting power of fake news: a big data analysis of the online media landscape from 2014 to 2016," New Media \& Society, vol. 20, no. 5, pp. 2028-2049, 2018.

[21] K. Sharma, F. Qian, H. Jiang, N. Ruchansky, M. Zhang, and Y. Liu, "Combating fake news," ACM Transactions on Intelligent Systems and Technology, vol. 10, no. 3, pp. 1-42, 2019.

[22] E. A. Pedersen, L. F. Loft, S. U. Jacobsen, B. Søborg, and J. Bigaard, "Strategic health communication on social media: Insights from a Danish social media campaign to address HPV vaccination hesitancy," Vaccine, vol. 38, no. 31, pp. 4909-4915, 2020.

[23] M. Wieringa, "What to account for when accounting for algorithms: a systematic literature review on algorithmic accountability," in ACM Conference on Fairness, Accountability, and Transparency (FAT), pp. 1-18, Barcelona, Spain, 2020.

[24] Y. Li, C. Su, J. Jia, Z. Xu, and R. Tian, "Analysis of development status of world artificial intelligence based on scientific measurement," Computer Science, vol. 44, no. 4, pp. 183-187, 2017.

[25] Y. Qian, Y. Liu, and Q. Z. Sheng, "Understanding hierarchical structural evolution in a scientific discipline: a case study of artificial intelligence," Journal of Informetrics, vol. 14, no. 3, article 101047, 2020. 